\title{
STABILITY ANALYSIS OF SSSS THIN RECTANGULAR PLATE USING MULTI - DEGREES OF FREEDOM TAYLOR MACLAURIN'S SERIES IN GALERKIN'S VARIATIONAL METHOD
}

\author{
N. N. Osadebe1, V. C. Nwokike ${ }^{2}$ and 0. A. Oguaghamba ${ }^{3,{ }^{*}}$ \\ 1,3 Department of Civil EngineERIng, University of Nigeria, NSUKKa, ENUGU State. NiGERIA

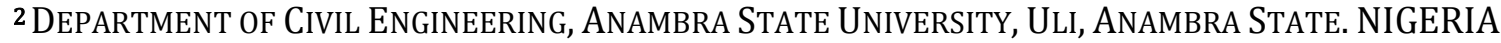 \\ E-mail addresses: ${ }^{1}$ mkemamaka@yahoo.com,2 vicnwokike@gmail.com, ${ }^{3}$ onyedikachi.oguaghamba@unn.edu.ng
}

\begin{abstract}
The stability analysis of all four edges simply supported (SSSS) thin rectangular plate using multi-degrees of freedom (MDOF) Taylor Maclaurin's series polynomial function in Galerkin's variational method has been investigated. This was achieved by truncating the two domain Taylor Maclaurin's series at the seventh term to evolve the general deflection polynomial function for thin rectangular plate continuum. Consequently, the SSSS plate boundary conditions were applied, reducing the polynomial function to four degrees of freedom function. Thereafter, Galerkin's model was applied to the classical governing differential equation of uniaxial plate buckling with the improved function to obtain the auxiliary equation, whose lowest eigenvalue corresponds to the SSSS plate buckling load coefficient, $K$. However, this process was facilitated using the commands in the Mathematica. The average percentage difference of $K$ - values from two previous works and the present study when compared with the exact solution stood at $0.066 \%, 0.011 \%$ and $0.002 \%$ respectively.This shows that MDOF function converges better than SDOF function. Among other revelations by the study is that Galerkin's variational methods remains a veritable tool for MDOF continuum problems.
\end{abstract}

Keywords: $\quad$ MDOF Polynomial Functions, SSSS Plate Boundary Conditions, Galerkin's Variational Method, Eigen value, Buckling Coefficients

\section{INTRODUCTION}

Thin plates are widely used as the main structural components of hull of ships, automotives, gate dams, aircrafts, bridges, etc., because of its two dimensional structural action. They also possess other interesting structural characteristics. For instance, when they are subjected to in plane loading (loading parallel to their fibre direction), they transit from their stable state of equilibrium to the unstable one, just like columns.
Such transition is normally referred to as buckling or structural instability. During this transition, a critical point exists where an infinitesimal increase in load can cause the plate surface to buckle. The load at this critical point defines the buckling strength of the plate, or the critical or buckling load. Increase in load beyond the load at the initiation of buckling increase the buckling deformations until collapse occurs [1].

\section{* Corresponding author Tel: + 234-806-086-9002}

This article was submitted to Nigerian Journal of Technology on January 29, 2016 by Engr. Vincent Maduakonam NWOKIKE who was on the verge of completing his doctoral studies at the Department of Civil Engineering, University of Nigeria, Nsukka. Unknown to the editorial office of NIJOTECH, Engr. Nwokike died in his sleep the same day this article was submitted. Until his demise, he was a Lecturer I at the Department of Civil Engineering, Odumeju Ojukwu University, Uli and hails from Adabebe, Amawbia, Awka South LGA of Anambra State, Nigeria.

You may recall that Prof. Nkemakonam Nwolisa Osadebe who was Nwokike's supervisor has earlier passed away on Friday October 23, 2015 (see Vol 35, Nos 1 and 2 of NIJOTECH). After peer review, the reviewers found the article publishable subject to some changes. The editorial board of NIJOTECH therefore appointed Dr. Onyedikachi Oguaghamba of Department of Civil Engineering, University of Nigeria, Nsukka to effect the changes on this paper, respond to reviewers comments and act as the new corresponding author to enable the journal publish this paper. 
So many researchers have investigated the buckling behaviour of thin rectangular plate subjected to uniform compression. Investigations involving simply supported plates subjected to uniform compression are common. Timoshenko and Gere [2] calculated the elastic buckling of simply supported plate, known as SSSS plate subjected to uniform compression by energy methods. Iyengar [3] used the Galerkin's method to analyse the elastic buckling the SSSS plate subjected to uniform uniaxial loads. These studies obtained the plate's critical load by assuming the deflection function of the plate in the form of truncated double Fourier series.

Ibearugbulem et.al [4] used truncated fourth order Taylor Maclaurin's series and formulated approximate single degree of freedom deflection function for the plate. They subsequently furnished the critical load of the plate by applying Ritz direct variational method. Ezeh et.al [5] used fourth order characteristic orthogonal polynomials against Taylor Maclaurin's series and obtained the deflection function for the the plate. Their deflection functions are identical to those of Ibearugbulem et. al [4]. Okafor and Udeh [6] toed the same path to obtain their deflection function for the plate. Rather than apply Ritz direct variational method,they used Galerkin's indirect variational method to obtainthe shear and bending stresses of the plate subjected to uniformly distributed lateral loads. The degree of freedom (DOF) of a continuum could be defined as the number of independent displacement admissible by the system in the course of its motion. Truncated fourth order Taylor Maclaurin's series or characteristic orthogonal polynomials for deflection solution in plates' analysis usually reduce to single degree of freedom Eigen - value problem. Whereas, the inclusion of fifth, sixth, seventh and other higher terms of the seriesor orthogonal polynomials would result to multi - degrees of freedom Eigen - value problem, whose final solution would improve convergence.

No researcher has attempted to use these higher order polynomials of Taylor Maclaurin's series to formulate the deflection function of simply supported plate. Perhaps, use the Galerkin's method to finally obtain the elastic buckling of the plate subjected to uniform compression. Rather, Ibearugbulem et. al [7] deposited that higher DOF does not improve convergence in plates' continuum analysis but would lead to rigorous work. They further concluded that Galerkin's method cannot be used for any MDOF polynomial function in plates' continuum analysis other than SDOF. In which case, they recommended only Ritz variational and Work principle approaches as the only method suitable for MDOF plates' continuum analysis.

This paper therefore, presents an improved Taylor Maclaurin's solution approach that considered higher order functions in the Galerkin' sindirect variational method towards establishing multi degrees of freedom eigen value problem of the elastic buckling of SSSS plate subjected to uniform uniaxial load along $\mathrm{x}$ direction. The fundamental theories applied are the Kirchhoff's plate theory, Galerkin's indirect variation method. Mathematica®, a Wolfram Research developed tool for doing mathematics on the computer and for reporting the results would be used. Mathematica ${ }^{\circledR}$ has numerical, graphical, and symbolic capabilities. Its basic features include arbitrary precision arithmetic; differential and integral calculus (routines for both symbolic and numerical evaluation); infinite and finite series, limits and products; expansion and factoring of algebraic expressions; linear algebra; solving systems of equations; and two and three - dimensional graphics. Mathematica $($ packages and custom tools include procedures for probability and statistics [8].

\section{CLASSICAL GOVERNING DIFFERENTIAL EQUATION OF BUCKLING PLATES' BUCKLING}

Figure 1 shows an isotropic elastic thin rectangular plate of length "a" and width " $b$ ". The plate is acted upon by axially compressed load in which, $\mathrm{N}_{\mathrm{x}}$ represents the magnitude of the compressive load.

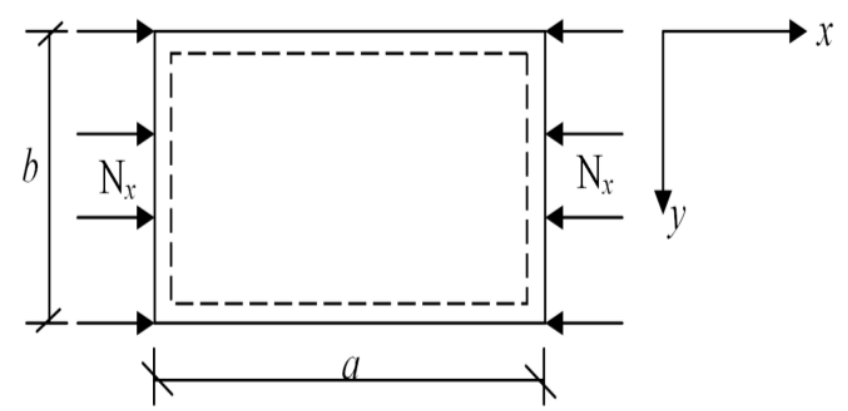

Figure 1: Analytical Model: Rectangular Thin Plate Element with Uniform Compression.

The equation that describes the behaviour of a thin elastic plate subjected to in plane load along $\mathrm{x}$ coordinate according to Kirchhoff's and Venant hypothesis is given by Ventsel and Krauthammer [9] as:

$$
\frac{\partial^{4} w}{\partial x^{4}}+2 \frac{\partial^{4} w}{\partial x^{2} \partial y^{2}}+\frac{\partial^{4} w}{\partial y^{4}}-\frac{N_{x}}{D} \frac{\partial^{2} w}{\partial x^{2}}=0
$$


In (1),

$$
D=\frac{E h^{3}}{12\left(1-\mu^{2}\right)}
$$

"E", "h" and " $\mu$ "are Young's of modulus of elasticity [1], thickness and Poisson's ratio of plate respectively; $\mathrm{N}_{\mathrm{x}}$ is the applied uniform compression along $\mathrm{x}-$ coordinate of the plate.

Expressing the independent coordinates $\mathrm{x}$ and $\mathrm{y}$ in the form of non-dimensional coordinates say $\mathrm{R}$ and $\mathrm{Q}$ for $\mathrm{x}$ and y directions respectively, as in [10].

That is:

$$
\begin{aligned}
& \mathrm{x}=\mathrm{aR} ; \quad 0 \leq R \leq 1 \\
& \mathrm{y}=\mathrm{bQ} \quad 0 \leq Q \leq 1
\end{aligned}
$$

where, $\mathrm{x}$ and $\mathrm{y}$ are the directional coordinates of the thin rectangular plate's surface along $a$ and $b$ directions respectively; $\mathrm{R}$ and $\mathrm{Q}$ are the corresponding non-dimensional surface or lateral dimensions of the plate along a and $\mathrm{b}$ directions respectively in the limits 0 to 1.0 .

Then, the derivatives in Equation (1) in nondimensional coordinates $\mathrm{R}$ and $\mathrm{Q}$ transforms as follows:

$$
\begin{array}{r}
\frac{\partial^{4} w}{\partial x^{4}}=\frac{1}{a^{4}} \frac{\partial^{4} w}{\partial R^{4}} ; \frac{\partial^{4} w}{\partial y^{4}}=\frac{1}{b^{4}} \frac{\partial^{4} w}{\partial Q^{4}} \\
\frac{\partial^{4} w}{\partial x^{2} \partial y^{2}}=\frac{1}{a^{2} b^{2}} \frac{\partial^{4} w}{\partial R^{2} \partial Q^{2}} ; \frac{\partial^{2} w}{\partial x^{2}}=\frac{\partial^{2} w}{a^{2} \partial R^{2}}
\end{array}
$$

Where, the aspect ratio, $\mathrm{p}$ and the plate's lateral dimensions, $a$ and $b$ is related as [10]:

$$
\mathrm{p}=\frac{\mathrm{a}}{\mathrm{b}} ; \mathrm{a}=\mathrm{pb}
$$

Thus, Equation (1) becomes:

$$
\begin{gathered}
\frac{1}{p^{4} b^{4}} \frac{\partial^{4} w}{\partial R^{4}}+2 \frac{\partial^{4} w}{p^{2} b^{4} \partial R^{2} \partial Q^{2}}+\frac{\partial^{4} w}{b^{4} \partial Q^{4}} \\
-\frac{N_{x}}{D p^{2} b^{2}} \frac{\partial}{\partial R}\left(\frac{\partial w}{\partial R}\right)=0 \\
\frac{1}{p^{2}} \frac{\partial^{4} w}{\partial R^{4}}+2 \frac{\partial^{4} w}{\partial R^{2} \partial Q^{2}}+\frac{P^{2} \partial^{4} w}{\partial Q^{4}}-\frac{N_{x} b^{2}}{D} \frac{\partial^{2} w}{\partial R^{2}}=0
\end{gathered}
$$

\section{MDOF POLYNOMIAL FUNCTION FOR SSSS THIN PLATE}

Ibearugbulem [4] used Taylor Maclaurin series as in Stroud[10] to derive the deflection polynomial function of a rectangular thin isotropic plate subjected to in-plane load in $\mathrm{x}$ - direction. The function which satisfies Equation (8) and approximately describes the deflection of the plate under uni-axial loading is given as:

$$
w=\sum_{m=o}^{\infty} \sum_{n=o}^{\infty} a_{m} R^{m} b_{n} \cdot Q^{n}
$$

Truncating Equation (9) at seventh term gave the deflection function for the plate as:

$$
\begin{aligned}
w=\left(a_{0}+a_{1} R\right. & +a_{2} R^{2}+a_{3} R^{3}+a_{4} R^{4}+a_{5} R^{5} \\
& \left.+a_{6} R^{6}+a_{7} R^{7}\right)\left(b_{0}+b_{1} Q+b_{2} Q^{2}\right. \\
& +b_{3} Q^{3}+b_{4} Q^{4}+b_{5} Q^{5} \\
& \left.+b_{6} Q^{6}+b_{7} Q^{7}\right)
\end{aligned}
$$

Where, $a_{m}$ and $b_{n}$ are the unknown coefficients which would be evaluated using the boundary conditions of the plate. $\mathrm{R}^{\mathrm{m}}$ and $\mathrm{Q}^{\mathrm{n}}$ are the non - dimensional directions of the plates corresponding to $\mathrm{x}$ and $\mathrm{y}$ directions respectively.

For SSSS thin rectangular plate, the prescribed boundary conditions along $\mathrm{R}$ and $\mathrm{Q}$ directions are given as:

Boundary Conditions along $\mathrm{R}$ - direction

$$
\begin{gathered}
{\left[\frac{\partial^{2} w}{\partial R^{2}}\right]_{R=0 \text { or } 1}=0} \\
{[w]_{R=0 \text { or } 1 \quad=0}}
\end{gathered}
$$

Boundary Conditions along $Q$ - direction

$$
\begin{aligned}
& {\left[\frac{\partial^{2} w}{\partial Q^{2}}\right]_{Q=0 \text { or } 1}=0} \\
& {[w]_{Q=0 \text { or } 1}=0}
\end{aligned}
$$

Implementing these boundary conditions (Equations 11, 12, 13 and 14) in Equation (10) gave the fourth degree of freedom deflection polynomial function as:

$$
\begin{aligned}
w=q_{1}\left(\mathrm{R}-2 \mathrm{R}^{3}\right. & \left.+\mathrm{R}^{4}\right)\left(\mathrm{Q}-2 \mathrm{Q}^{3}+\mathrm{Q}^{4}\right) \\
& +q_{2}\left(7 \mathrm{R}-10 \mathrm{R}^{3}+3 \mathrm{R}^{5}\right)\left(7 \mathrm{Q}-10 \mathrm{Q}^{3}\right. \\
& \left.+3 \mathrm{Q}^{5}\right) \\
& +q_{3}\left(4 \mathrm{R}-5 \mathrm{R}^{3}+\mathrm{R}^{6}\right)\left(4 \mathrm{Q}-5 \mathrm{Q}^{3}+\mathrm{Q}^{6}\right) \\
& +q_{4}\left(6 \mathrm{R}-7 \mathrm{R}^{3}+\mathrm{R}^{7}\right)\left(6 \mathrm{Q}-7 \mathrm{Q}^{3}\right. \\
& \left.+\mathrm{Q}^{7}\right) \\
w & =w_{1}+w_{2}+w_{3}+w_{4}
\end{aligned}
$$

Where, $q_{1}=a_{4} b_{4} ; q_{2}=\frac{a_{5} b_{5}}{9} ; q_{3}=a_{6} b_{6} ; q_{4}=a_{7} b_{7}$.

They are the independent coefficients of the respective independent displacement functions of the four DOF polynomial. Previous researchers have always presumed the SSSS thin rectangular plate to be executing mostly one independent displacement, which is not so correct. Hence, such erroneous presumptions would no doubt lead to nearly false impression of plates' continuum problem. Even worse where the problem statement bothers on stability problems, such eigenvalues may possess some danger to the structure under consideration.

\section{APPLICATION OF THE GALERKIN'S VARIATIONAL METHOD}

Ventsel and Krauthammer [9] gave the classical Galerkin's generalized of solution of differential 
equation for any given 2D boundary value elastostatic problems in non-dimensional coordinates $\mathrm{R}$ and $\mathrm{Q}$ domain as:

$$
a_{i j}=\iint_{A}[L(w)-p] f(R, Q) d R d Q=0
$$

Where, $\mathrm{p}$ and $\mathrm{L}(\mathrm{w})$ are the applied load and resistance offered by the system respectively. Both of them are represented by the left hand side of Equation (8), $a_{i j}$ is the residual error; while $f(R, Q)$ is the non-coefficient shape function along R-Q directions in the plate's domain $\mathrm{a}$ and $\mathrm{b}$ obtained from Equations (15) and (16) given as:

$$
\begin{array}{r}
f(R, Q)=\sum_{j=1}^{4} f_{j}(R, Q)=\sum_{j=1}^{4} \frac{w_{j}(R, Q)}{q_{j}}=f_{1}+f_{2}+f_{3}+f_{4} \\
f(R, Q)=\left(\mathrm{R}-2 \mathrm{R}^{3}+\mathrm{R}^{4}\right)\left(\mathrm{Q}-2 \mathrm{Q}^{3}+\mathrm{Q}^{4}\right)+\left(7 \mathrm{R}-10 \mathrm{R}^{3}+3 \mathrm{R}^{5}\right)\left(7 \mathrm{Q}-10 \mathrm{Q}^{3}+3 \mathrm{Q}^{5}\right) \\
+\left(4 \mathrm{R}-5 \mathrm{R}^{3}+\mathrm{R}^{6}\right)\left(4 \mathrm{Q}-5 \mathrm{Q}^{3}+\mathrm{Q}^{6}\right)+\left(6 \mathrm{R}-7 \mathrm{R}^{3}+\mathrm{R}^{7}\right)\left(6 \mathrm{Q}-7 \mathrm{Q}^{3}+\mathrm{Q}^{7}\right)
\end{array}
$$

Where, $w_{j}$ and $q_{j}$ represent the independent deflection function and its respective undetermined coefficient corresponding to jth degree of freedom of the plate.

Adopting the Galerkin's formulation in Equation (17) for SSSS plate stability problem in non - dimensional directions, R and Q with the fourth DOF deflection function in Equations (15) and (16) gave:

$$
\begin{gathered}
\mathrm{a}_{\mathrm{ij}}=\iint_{00}^{11}\left(\frac{1}{p^{2}} \frac{\partial^{4} w}{\partial R^{4}}+2 \frac{\partial^{4} w}{\partial R^{2} \partial Q^{2}}+\frac{P^{2} \partial^{4} w}{\partial Q^{4}}-\frac{N_{x} b^{2}}{D} \frac{\partial^{2} w}{\partial R^{2}}\right) f(R, Q) \partial \mathrm{R} \partial \mathrm{Q}=0 \\
\mathrm{a}_{\mathrm{ij}}=\iint_{00}^{11}\left(\frac{1}{p^{2}} \frac{\partial^{4} w}{\partial R^{4}}+2 \frac{\partial^{4} w}{\partial R^{2} \partial Q^{2}}+\frac{P^{2} \partial^{4} w}{\partial Q^{4}}\right) f(R, Q) \partial \mathrm{R} \partial \mathrm{Q}=\int_{0}^{1} \int_{0}^{1} \frac{N_{x} b^{2}}{D} \frac{\partial^{2} w}{\partial R^{2}} f(R, Q) \partial \mathrm{R} \partial \mathrm{Q}
\end{gathered}
$$

where,

$$
\begin{gathered}
\frac{\partial^{2} w}{\partial R^{2}}=q_{1}\left(-12 \mathrm{R}+12 \mathrm{R}^{2}\right)\left(\mathrm{Q}-2 \mathrm{Q}^{3}+\mathrm{Q}^{4}\right)+q_{2}\left(-60 \mathrm{R}+60 \mathrm{R}^{3}\right)\left(7 \mathrm{Q}-10 \mathrm{Q}^{3}+3 \mathrm{Q}^{5}\right)+ \\
q_{3}\left(-30 \mathrm{R}+30 \mathrm{R}^{4}\right)\left(4 \mathrm{Q}-5 \mathrm{Q}^{3}+\mathrm{Q}^{6}\right)+q_{4}\left(-42 \mathrm{R}+42 \mathrm{R}^{5}\right)\left(6 \mathrm{Q}-7 \mathrm{Q}^{3}+\mathrm{Q}^{7}\right) \\
\frac{\partial^{4} w}{\partial R^{4}}=24 q_{1}\left(\mathrm{Q}-2 \mathrm{Q}^{3}+\mathrm{Q}^{4}\right)+360 q_{2} R\left(7 \mathrm{Q}-10 \mathrm{Q}^{3}+3 \mathrm{Q}^{5}\right)+360 q_{3} R^{2}\left(4 \mathrm{Q}-5 \mathrm{Q}^{3}+\mathrm{Q}^{6}\right)+ \\
840 q_{4} R^{3}\left(6 \mathrm{Q}-7 \mathrm{Q}^{3}+\mathrm{Q}^{7}\right) \\
\frac{\partial^{4} w}{\partial Q^{4}}=24 q_{1}\left(\mathrm{R}-2 \mathrm{R}^{3}+\mathrm{R}^{4}\right)+360 q_{2}\left(7 \mathrm{R}-10 \mathrm{R}^{3}+3 \mathrm{R}^{5}\right) Q+360 q_{3}\left(4 \mathrm{R}-5 \mathrm{R}^{3}+\mathrm{R}^{6}\right) Q^{2}+ \\
840 q_{4}\left(6 \mathrm{R}-7 \mathrm{R}^{3}+\mathrm{R}^{7}\right) Q^{3} \\
\frac{\partial^{4} w}{\partial R^{2} \partial Q^{2}}=q_{1}\left(-12 \mathrm{R}+12 \mathrm{R}^{2}\right)\left(-12 \mathrm{Q}+12 \mathrm{Q}^{2}\right)+q_{2}\left(-60 \mathrm{R}+60 \mathrm{R}^{3}\right)\left(-60 \mathrm{Q}+60 \mathrm{Q}^{3}\right)+ \\
q_{3}\left(-30 \mathrm{R}+30 \mathrm{R}^{4}\right)\left(-30 \mathrm{Q}+30 \mathrm{Q}^{4}\right)+q_{4}\left(-42 \mathrm{R}+42 \mathrm{R}^{5}\right)\left(-42 \mathrm{Q}+42 \mathrm{Q}^{5}\right)
\end{gathered}
$$

Thus,

$$
\begin{gathered}
\mathrm{a}_{\mathrm{ij}}=\int_{0}^{1} \int_{0}^{1}\left(\frac { 1 } { p ^ { 2 } } \left[24 q_{1}\left(\mathrm{Q}-2 \mathrm{Q}^{3}+\mathrm{Q}^{4}\right)+360 q_{2} R\left(7 \mathrm{Q}-10 \mathrm{Q}^{3}+3 \mathrm{Q}^{5}\right)+360 q_{3} R^{2}\left(4 \mathrm{Q}-5 \mathrm{Q}^{3}+\right.\right.\right. \\
\left.\left.\mathrm{Q}^{6}\right)+840 q_{4} R^{3}\left(6 \mathrm{Q}-7 \mathrm{Q}^{3}+\mathrm{Q}^{7}\right)\right]+2\left[q_{1}\left(-12 \mathrm{R}+12 \mathrm{R}^{2}\right)\left(-12 \mathrm{Q}+12 \mathrm{Q}^{2}\right)+\right. \\
q_{2}\left(-60 \mathrm{R}+60 \mathrm{R}^{3}\right)\left(-60 \mathrm{Q}+60 \mathrm{Q}^{3}\right)+q_{3}\left(-30 \mathrm{R}+30 \mathrm{R}^{4}\right)\left(-30 \mathrm{Q}+30 \mathrm{Q}^{4}\right)+ \\
\left.q_{4}\left(-42 \mathrm{R}+42 \mathrm{R}^{5}\right)\left(-42 \mathrm{Q}+42 \mathrm{Q}^{5}\right)\right]+P^{2}\left[24 q_{1}\left(\mathrm{R}-2 \mathrm{R}^{3}+\mathrm{R}^{4}\right)+360 q_{2}\left(7 \mathrm{R}-10 \mathrm{R}^{3}+3 \mathrm{R}^{5}\right) Q\right. \\
\left.\left.+360 q_{3}\left(4 \mathrm{R}-5 \mathrm{R}^{3}+\mathrm{R}^{6}\right) Q^{2}+840 q_{4}\left(6 \mathrm{R}-7 \mathrm{R}^{3}+\mathrm{R}^{7}\right) Q^{3}\right]\right)\left(\left(\mathrm{R}-2 \mathrm{R}^{3}+\mathrm{R}^{4}\right)\left(\mathrm{Q}-2 \mathrm{Q}^{3}+\mathrm{Q}^{4}\right)\right. \\
+\left(7 \mathrm{R}-10 \mathrm{R}^{3}+3 \mathrm{R}^{5}\right)\left(7 \mathrm{Q}-10 \mathrm{Q}^{3}+3 \mathrm{Q}^{5}\right)+\left(4 \mathrm{R}-5 \mathrm{R}^{3}+\mathrm{R}^{6}\right)\left(4 \mathrm{Q}-5 \mathrm{Q}^{3}+\mathrm{Q}^{6}\right)+ \\
\left.\left(6 \mathrm{R}-7 \mathrm{R}^{3}+\mathrm{R}^{7}\right)\left(6 \mathrm{Q}-7 \mathrm{Q}^{3}+\mathrm{Q}^{7}\right)\right) \partial \mathrm{R} \partial \mathrm{Q}
\end{gathered}
$$




$$
\begin{aligned}
=\frac{N_{x} b^{2}}{D} \int_{0}^{1} \int_{0}^{1}\left(q_{1}\right. & \left.-12 \mathrm{R}+12 \mathrm{R}^{2}\right)\left(\mathrm{Q}-2 \mathrm{Q}^{3}+\mathrm{Q}^{4}\right)+q_{2}\left(-60 \mathrm{R}+60 \mathrm{R}^{3}\right)\left(7 \mathrm{Q}-10 \mathrm{Q}^{3}+3 \mathrm{Q}^{5}\right)+q_{3}\left(-30 \mathrm{R}+30 \mathrm{R}^{4}\right)(4 \mathrm{Q} \\
& \left.\left.-5 \mathrm{Q}^{3}+\mathrm{Q}^{6}\right)+q_{4}\left(-42 \mathrm{R}+42 \mathrm{R}^{5}\right)\left(6 \mathrm{Q}-7 \mathrm{Q}^{3}+\mathrm{Q}^{7}\right)\right)\left(\left(\mathrm{R}-2 \mathrm{R}^{3}+\mathrm{R}^{4}\right)\left(\mathrm{Q}-2 \mathrm{Q}^{3}+\mathrm{Q}^{4}\right)\right. \\
& +\left(7 \mathrm{R}-10 \mathrm{R}^{3}+3 \mathrm{R}^{5}\right)\left(7 \mathrm{Q}-10 \mathrm{Q}^{3}+3 \mathrm{Q}^{5}\right)+\left(4 \mathrm{R}-5 \mathrm{R}^{3}+\mathrm{R}^{6}\right) \times\left(4 \mathrm{Q}-5 \mathrm{Q}^{3}+\mathrm{Q}^{6}\right) \\
& \left.+\left(6 \mathrm{R}-7 \mathrm{R}^{3}+\mathrm{R}^{7}\right)\left(6 \mathrm{Q}-7 \mathrm{Q}^{3}+\mathrm{Q}^{7}\right)\right) \partial \mathrm{R} \partial \mathrm{Q}
\end{aligned}
$$

Replacing the integral in Equation (20) by the sum of integral, we obtained the following system of linear algebraic equations:

$$
\begin{gathered}
\alpha_{11} q_{1}+\alpha_{12} q_{2}+\alpha_{13} q_{3}+\alpha_{14} q_{4}=\frac{N_{x} b^{2}}{D}\left(\beta_{11} q_{1}+\beta_{12} q_{2}+\beta_{13} q_{3}+\beta_{14} q_{4}\right) \\
\alpha_{21} q_{1}+\alpha_{22} q_{2}+\alpha_{23} q_{3}+\alpha_{24} q_{4}=\frac{N_{x} b^{2}}{D}\left(\beta_{21} q_{1}+\beta_{22} q_{2}+\beta_{23} q_{3}+\beta_{24} q_{4}\right) \\
\alpha_{31} q_{1}+\alpha_{32} q_{2}+\alpha_{33} q_{3}+\alpha_{34} q_{4}=\frac{N_{x} b^{2}}{D}\left(\beta_{31} q_{1}+\beta_{32} q_{2}+\beta_{33} q_{3}+\beta_{34} q_{4}\right) \\
\alpha_{41} q_{1}+\alpha_{42} q_{2}+\alpha_{43} q_{3}+\alpha_{44} q_{4}=\frac{N_{x} b^{2}}{D}\left(\beta_{41} q_{1}+\beta_{42} q_{2}+\beta_{43} q_{3}+\beta_{44} q_{4}\right)
\end{gathered}
$$

Expressing Equation (21) in the canonical matrix form, we have:

$$
\begin{aligned}
& \left(\alpha_{11}-\mathrm{K} \beta_{11}\right) q_{1}+\left(\alpha_{12}-\mathrm{K} \beta_{12}\right) q_{2}+\left(\alpha_{13}-\mathrm{K} \beta_{13}\right) q_{3}+\left(\alpha_{14}-\mathrm{K} \beta_{14}\right) q_{4}=0 \\
& \left(\alpha_{21}-\mathrm{K} \beta_{21}\right) q_{1}+\left(\alpha_{22}-\mathrm{K} \beta_{22}\right) q_{2}+\left(\alpha_{23}-\mathrm{K} \beta_{23}\right) q_{3}+\left(\alpha_{24}-\mathrm{K} \beta_{24}\right) q_{4}=0 \\
& \left(\alpha_{31}-\mathrm{K} \beta_{31}\right) q_{1}+\left(\alpha_{32}-\mathrm{K} \beta_{32}\right) q_{2}+\left(\alpha_{33}-\mathrm{K} \beta_{33}\right) q_{3}+\left(\alpha_{34}-\mathrm{K} \beta_{34}\right) q_{4}=0 \\
& \left(\alpha_{41}-\mathrm{K} \beta_{41}\right) q_{1}+\left(\alpha_{42}-\mathrm{K} \beta_{42}\right) q_{2}+\left(\alpha_{43}-\mathrm{K} \beta_{43}\right) q_{3}+\left(\alpha_{44}-\mathrm{K} \beta_{44}\right) q_{4}=0 \\
& {\left[\begin{array}{lll}
\left(\alpha_{11}-\mathrm{K} \beta_{11}\right) & \left(\alpha_{12}-\mathrm{K} \beta_{12}\right)\left(\alpha_{13}-\mathrm{K} \beta_{13}\right) & \left(\alpha_{14}-\mathrm{K} \beta_{14}\right) \\
\left(\alpha_{21}-\mathrm{K} \beta_{21}\right) & \left(\alpha_{22}-\mathrm{K} \beta_{22}\right)\left(\alpha_{23}-\mathrm{K} \beta_{23}\right) & \left(\alpha_{24}-\mathrm{K} \beta_{24}\right) \\
\left(\alpha_{31}-\mathrm{K} \beta_{31}\right) & \left(\alpha_{32}-\mathrm{K} \beta_{32}\right)\left(\alpha_{33}-\mathrm{K} \beta_{33}\right) & \left(\alpha_{34}-\mathrm{K} \beta_{34}\right) \\
\left(\alpha_{41}-\mathrm{K} \beta_{41}\right) & \left(\alpha_{42}-\mathrm{K} \beta_{42}\right)\left(\alpha_{43}-\mathrm{K} \beta_{43}\right) & \left(\alpha_{44}-\mathrm{K} \beta_{44}\right)
\end{array}\right]\left[\begin{array}{l}
q_{1} \\
q_{2} \\
q_{3} \\
q_{4}
\end{array}\right]=0} \\
& \text { where, } \mathrm{K}=\frac{\mathrm{N}_{\mathrm{x}} \mathrm{b}^{2}}{\mathrm{D}} ; \alpha_{i j}=\iint_{00}^{11}\left(\frac{1}{p^{2}} \frac{\partial^{4} w}{\partial R^{4}}+2 \frac{\partial^{4} w}{\partial R^{2} \partial Q^{2}}+\frac{P^{2} \partial^{4} w}{\partial Q^{4}}\right) f_{j}(R, Q) \partial \mathrm{R} \partial \mathrm{Q} ; \mathrm{j}=1,2,3,4 \text { for } \mathrm{i}=1 \text { to } 4 \\
& \text { and } \beta_{i j}=\iint_{00}^{11}\left(\frac{\partial^{2} w}{\partial R^{2}}\right) f_{j}(R, Q) \partial \mathrm{R} \partial \mathrm{Q} ; \quad \mathrm{j}=1,2,3,4 \text { for } \mathrm{i}=1 \text { to } 4
\end{aligned}
$$

For non-trivial solution, the eigenvector, $\mathrm{q}_{\mathrm{i}}$ in Equation (22) cannot be zero but the Eigen-value.

That is,

$$
\left[\begin{array}{l}
q_{1} \\
q_{2} \\
q_{3} \\
q_{4}
\end{array}\right] \neq 0 ;\left[\begin{array}{lll}
\left(\alpha_{11}-\mathrm{K} \beta_{11}\right) & \left(\alpha_{12}-\mathrm{K} \beta_{12}\right)\left(\alpha_{13}-\mathrm{K} \beta_{13}\right) & \left(\alpha_{14}-\mathrm{K} \beta_{14}\right) \\
\left(\alpha_{21}-\mathrm{K} \beta_{21}\right) & \left(\alpha_{22}-\mathrm{K} \beta_{22}\right)\left(\alpha_{23}-\mathrm{K} \beta_{23}\right) & \left(\alpha_{24}-\mathrm{K} \beta_{24}\right) \\
\left(\alpha_{31}-\mathrm{K} \beta_{31}\right) & \left(\alpha_{32}-\mathrm{K} \beta_{32}\right)\left(\alpha_{33}-\mathrm{K} \beta_{33}\right) & \left(\alpha_{34}-\mathrm{K} \beta_{34}\right) \\
\left(\alpha_{41}-\mathrm{K} \beta_{41}\right) & \left(\alpha_{42}-\mathrm{K} \beta_{42}\right)\left(\alpha_{43}-\mathrm{K} \beta_{43}\right) & \left(\alpha_{44}-\mathrm{K} \beta_{44}\right)
\end{array}\right]=0
$$

Hence, the determinant of the scalar matrix results to the auxiliary equation as follows:

$$
\left|\begin{array}{lll}
\left(\alpha_{11}-\mathrm{K} \beta_{11}\right) & \left(\alpha_{12}-\mathrm{K} \beta_{12}\right)\left(\alpha_{13}-\mathrm{K} \beta_{13}\right) & \left(\alpha_{14}-\mathrm{K} \beta_{14}\right) \\
\left(\alpha_{21}-\mathrm{K} \beta_{21}\right) & \left(\alpha_{22}-\mathrm{K} \beta_{22}\right)\left(\alpha_{23}-\mathrm{K} \beta_{23}\right) & \left(\alpha_{24}-\mathrm{K} \beta_{24}\right) \\
\left(\alpha_{31}-\mathrm{K} \beta_{31}\right) & \left(\alpha_{32}-\mathrm{K} \beta_{32}\right)\left(\alpha_{33}-\mathrm{K} \beta_{33}\right) & \left(\alpha_{34}-\mathrm{K} \beta_{34}\right) \\
\left(\alpha_{41}-\mathrm{K} \beta_{41}\right) & \left(\alpha_{42}-\mathrm{K} \beta_{42}\right)\left(\alpha_{43}-\mathrm{K} \beta_{43}\right) & \left(\alpha_{44}-\mathrm{K} \beta_{44}\right)
\end{array}\right|=0
$$

This auxiliary equation is also a fourth degree single polynomial whose lowest eigenvalue corresponds to the lowest load level for which the critical load exists. The polynomial may be written as:

$$
\mathrm{C}_{0}+\mathrm{C}_{1}+\mathrm{C}_{2} \mathrm{~K}^{2}+\mathrm{C}_{3} \mathrm{~K}^{3}+\mathrm{C}_{4} \mathrm{~K}^{4}=0
$$

In (24), $C_{i}$ is a coefficient proportionality dependent of the aspect ratio, $\mathrm{p}$ of the plate. Equation (24) was solved at

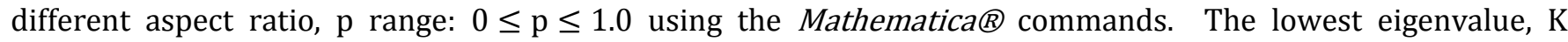
obtained for the various aspect ratios corresponds to the lowest load level for which the critical buckling exists. The lowest eigenvalue, K results from this study, those of Iyengar [3], Ibearugbulem et. al [4] and Ezeh et. al [5] are shown in Table 1.0. The percentage differences (error) between this study, Ibearugbulem et. al and Ezeh et. al with of those of Iyengar are also shown in Table 1.0. Where the \% error is calculated as in Shimpi and Patel [9] as: 
Stability Analysis Of SSSS Thin Rectangular Plate USing Mdof Taylor Maclaurin’s Series ... N. N. Osadebe, et al

$$
\text { Percentage Error }=\left(\frac{\text { Value obtained by a theory }}{\text { corresponding value by exact theory }}-1\right) \times 100
$$

Table 1: $K$ values for different aspect ratios for the buckling of SS-SS thin plate

\begin{tabular}{|c|c|c|c|c|c|c|c|}
\hline \multirow[b]{2}{*}{$\begin{array}{l}\text { Aspect } \\
\text { ratio, } P\end{array}$} & \multicolumn{7}{|c|}{$\begin{array}{c}\text { Buckling load coefficients, K for SSS thin rectangular plates at different aspect ratios, P by researchers and } \\
\text { present study }\end{array}$} \\
\hline & [3] & [4] & [5] & $\begin{array}{l}\text { Present } \\
\text { study }\end{array}$ & $\begin{array}{l}\% \text { Error between [3] } \\
\text { and Present study }\end{array}$ & $\begin{array}{l}\text { \% Error between } \\
{[3] \text { and }[4]}\end{array}$ & $\begin{array}{c}\% \text { Error } \\
\text { between [3] and } \\
{[5]}\end{array}$ \\
\hline 0.1 & 102.010 & 102.110 & 102.059 & 102.011 & 0.001 & 0.098 & 0.048 \\
\hline 0.2 & 27.040 & 27.065 & 27.051 & 27.045 & 0.018 & 0.092 & 0.041 \\
\hline 0.3 & 13.201 & 13.212 & 13.205 & 13.201 & 0.000 & 0.083 & 0.030 \\
\hline 0.4 & 8.410 & 8.416 & 8.412 & 8.410 & 0.000 & 0.071 & 0.024 \\
\hline 0.5 & 6.250 & 6.254 & 6.251 & 6.250 & 0.000 & 0.064 & 0.016 \\
\hline 0.6 & 5.138 & 5.141 & 5.138 & 5.138 & 0.000 & 0.058 & 0.000 \\
\hline 0.7 & 4.531 & 4.533 & 4.531 & 4.531 & 0.000 & 0.044 & 0.000 \\
\hline 0.8 & 4.203 & 4.205 & 4.202 & 4.203 & 0.000 & 0.048 & -0.024 \\
\hline 0.9 & 4.045 & 4.047 & 4.044 & 4.045 & 0.000 & 0.049 & -0.025 \\
\hline 1.0 & 4.000 & 4.002 & 4.000 & 4.000 & 0.000 & 0.050 & 0.000 \\
\hline \multicolumn{5}{|c|}{ Average \% Error } & 0.002 & 0.066 & 0.011 \\
\hline
\end{tabular}

\section{RESULTS AND DISCUSSION}

The results for the critical buckling load coefficients at aspect ratios, $p$ range: $(0.1 \geq P \geq 1.0)$ from the present study and previous works are presented in Table 1.0. A close observation of the table revealed that the results obtained using four degrees of freedom deflection polynomial function of the Taylor Maclaurin's series converges almost to those of the exact solution by Iyengar [3].

The average percentage difference between the solution from Iyengar [3] and Ibearugbulem et. al [4]; Ezeh et. al [5] and Iyengar [3] and the present study and Iyengar [3] are $0.066 \%, 0.011 \%$ and $0.002 \%$. This reveals clearly the improved convergence occasioned by conceiving the plate's continuum to be executing multiple degrees of freedom under any form of loading. This absolutely invalidates and clearly disprove the earlier supposition by Ibearugbulem et. al [7] that convergence in line and plates' continuum does not improve by increasing the number of terms in the polynomial series beyond the term that contains the fourth power. It has on the other hand substantiated Galerkin's [13] claim that his solution approaches the exact solution, if the DOF of the polynomial function tends to infinity. In this way, the continuum under consideration would be analysed as a continuous system (infinite DOF system), which is actually the case, rather than assuming single of lumped (finite) DOF system which been the trend by past researchers.

\section{CONCLUSION}

The effect of uniaxial in plane load on the elastic thin rectangular plate with SSSS boundary conditions has been investigated using higher degrees of freedom polynomial. The successful application of the four degrees of freedom polynomial function in the Galerkin's method for buckling analysis of SSSS plate has also put to rest the recent conjecture of Ibearugbulem et. al [7] that Galerkin's method could be constrained for the analysis of continuum with multi-degrees of freedom.

The following inferences are therefore adduced:

a) Truncation of Taylor Maclaurin's series beyond fourth term would result to higher degrees of freedoms polynomial functions.

b) Convergence of Taylor Maclaurin's series are improved with higher degrees of freedoms polynomial functions.

c) Four DOF polynomial function for SSSS plate's buckling approaches the exact solution better than one DOF polynomial function.

d) Galerkin's method remains suitable for the analysis multi degrees of freedom continuum.

e) Application of Galerkin's method for plate continuum could be made easier and faster by the use of commands in the Mathematica $($.

\section{REFERENCES}

[1] Bleich, F. Buckling Strength of Metal Structures, McGraw-Hill, New York, 1952.

[2] Timoshenko, S. P. and Gere, J. M. Theory of Elastic Stability, McGraw-Hill, New York, 1961. 
[3] Iyengar, N. G. Structural Stability of Columns and Plates, Ellis Horwood Limited, New York, 1988.

[4] Ibearugbulem, O. M. Osadebe, N. N. Ezeh J. C. and Onwuka, D. O. "Buckling Analysis of Axially Compressed SSSS Thin Rectangular Plate using Taylor-Mclaurin shape function," International journal of civil and structural engineering, Vol. 2, No. 2, pp. 667 - 672, 2011.

[5] Ezeh, J. C., Ibearugbulem, M. O., Opara, H. E. and Oguaghamba, O. A. "Characteristics Orthogonal Polynomial Application to Galerkin's Indirect Variational Method for Simply Supported Plate under Inplane Loading," International Journal of Research in Engineering and Technology, Vol. 3, No. 4, pp. $720-725,2014$

[6] Okafor, F. O. and Udeh, O. T. "Direct Method of Analysis of an Isotropic Rectangular Plate using Characteristic Orthogonal Polynomials," Nigerian Journal of Technology, Vol. 34, No. 2, pp. 232 - 239, 2015.

[7] Ibearugbulem, O. M., Oguaghamba, O. A., Njoku K. O. and Nwaokeorie, M. "Using Line Continuum to Explain Work Principle Method for Structural Continuum Analysis," International Journal of
Innovative Research \& Development, Vol. 3, No. 9, pp. 365 - 370, 2014.

[8] Wolfram, S. Mathematica: A System for Doing Mathematics by Computer, Addison-Wesley, Redwood City, 1991.

[9] Ventsel, E. and Krauthammer, T. Thin Plates and Shells: Theory, Analysis and Applications, Maxwell Publishers Inc, New York, 2001.

[10] Oguaghamba, 0. A. "Analysis of Buckling and Postbuckling Loads of Isotropic Thin Rectangular Plates," Ph.D. Thesis Submitted to Postgraduate School, Federal University of Technology, Owerri, 2015.

[11] Stroud, K. A. Advanced engineering mathematics, Palgrave Macmillan, New York, 2003.

[12] Galerkin, B. G. "Series - solutions of some cases of equilibrium of elastic beams and plates," VestnInshenernov, Vol. 1, pp. 897 - 903, 1915.

[13] Shimpi, R. P. and Patel, H. G. "A two variable refined plate theory for orthotropic plate analysis," International Journal of Solids and Structures, Vol. 43, No. 22 - 23, pp. 6783 - 6799, 2006. 